\title{
Medical errors during COVID-19 pandemic: the role of emergency medicine
}

\author{
Shaghayegh Rahmani ${ }^{*}$, Kosar Deldar ${ }^{2}$, Sara Hemati Ali ${ }^{3}$ \\ 'Innovated Medical Research Center, Faculty of Medicine, Mashhad Branch, Islamic Azad University, Mashhad, Iran \\ ${ }^{2}$ Department of Emergency Medicine, Faculty of Medicine, North Khorasan University of Medical sciences, Bojnourd, Iran \\ ${ }^{3}$ Department of Emergency Medicine, Faculty of Medicine, Mashhad University of Medical sciences, Mashhad, Iran
}

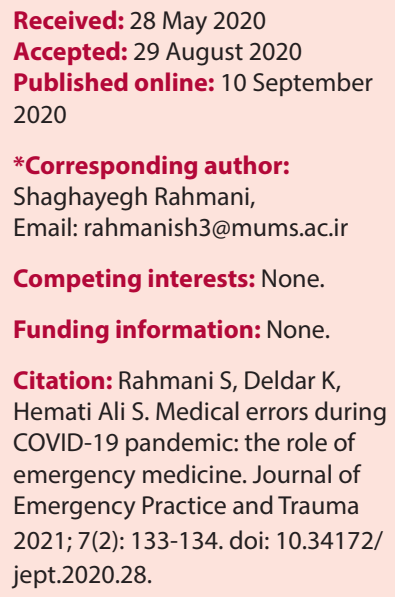

\begin{abstract}
Objective: Nowadays, many countries all over the world are involved with COVID-19 and the number of new cases and deaths are on a rise. The role of emergency medicine and physician-led triage is important in this period. We report some near missed cases in our academic center related to this pandemic.

Case Presentation: We report 5 cases that missed triage or received delayed diagnosis because of COVID-19 suspicion. Some cases are life threatening.

Conclusion: Although COVID-19 is the main health concern these days, other critical conditions should be considered. Stabilizing patients before transferring them between hospitals should be the essential goal of emergency department whether the patient is Corona virus infected or not. And before any intervention, the safety of healthcare workers must be ensured.
\end{abstract}

Keywords: COVID-19, Medical error, Emergency medicine

\section{Introduction}

It is about three months from the first reported case of COVID-19 in China and after a while, the World Health Organization (WHO) declared it as a global health concern and it became a pandemic (1). Nowadays, many countries all over the world are involved and the number of new cases and deaths are increasing (2). This pandemic cannot be compared with previous ones because of the social media and promoting public knowledge. This awareness has its Pros and Cons. People with any signs and symptoms rush into emergency departments (EDs) to be evaluated as suspicious cases of COVID-19. In this situation, triage staffs have a difficult time to separate different cases and identify the main patients' complaints (3).

As we mentioned before in our previous paper, although the number of cases other than COVID-19 has decreased significantly these days, some EDs might be at the risk of miss management (4). Shortage of personal protective equipment (PPE) and overcrowding of EDs are two important influential factors in miss-triage of patients in this pandemic (5).

One of the main differences between this pandemic and the previous ones is the presence of an emergency medicine specialist in EDs. Emergency medicine science is added to medicine to reduce medical errors. These physicians are trained to perform an extensive and detailed assessment of patients as soon as possible. The role of emergency medicine and physician-led triage is important in this period. We believe that the recruitment of an emergency medicine specialist in corona centers helps to reduce the number of medical errors and missed ED diagnoses. We report some cases of near missed cases in our academic center.

Case 1

A middle-aged male patient referred to our hospital with dyspnea. The emergency medical services (EMS) called the emergency medicine physician to refer him to the corona center. After tacking a brief history, it was found that he had chest pain, dyspnea and history of diabetes mellitus. Electrocardiogram confirmed ST elevation myocardial infarction and patient was transferred to the cardiology unit immediately.

Case 2

A young female with respiratory distress and fever referred to the ED. The triage nurse asked EMS to transfer her to a corona center. It was at the end of the shift of the emergency medicine specialist, when she inadvertently 
ran into the patient and visited her. Based on her ill appearance, the emergency medicine specialist requested to transfer the patient to cardiopulmonary resuscitation room. In primary evaluation, high blood sugar was reported and arterial blood gas and intravenous fluid therapy were done for the patient. Patient was admitted as diabetic ketoacidosis in the intensive care unit (ICU).

\section{Case 3}

A 67-year old opium addicted woman with fever, dyspnea and confusion referred to a corona center hospital after respiratory triage due to pin-point pupils and history of opium overdose. After securing airway and intravenous fluid infusion, naloxone was tried and before evaluating the response of the patient, she was transferred for performing pulmonary high-resolution computed tomography to rule out COVID-19. Also, she underwent brain computed tomography scan (CT) because of confusion. After negative radiologic findings for COVID-19, she was transferred to an internal medicine center and she was treated as an opium overdose patient with aspiration pneumonia.

\section{Case 4}

A 60-year old woman with the history of on and off abdominal pain with fever and occasionally dry cough referred to a COVID center. After respiratory triage due to normal chest $\mathrm{x}$-ray and patient reevaluation, positive murphy sign was found in abdominal examination. The patient was referred to another hospital for further investigation and this made the patient and her family confused and disappointed about the health care system.

\section{Case 5}

A middle aged male with a history of chronic obstructive pulmonary disease (COPD) was sitting in the triage room and requested to see the doctor. Because of low SPO2, our staff asked him to go to a corona center for consultation. With the patient's insistence, triage nurse asked the physician to visit the patient. His chief compliant was urinary retention and he had no signs and symptoms of respiratory disease or COPD exacerbation. He was an oxygen-dependent COPD case.

\section{Discussion}

The current outbreak of the COVID-19 in our country is a challenging issue for our ED staffs. EDs encounter elderly patients with multiple chronic conditions and complaints. Finding the exact cause of ED visits is hard and impossible in some cases (3). While the number of COVID-19 cases in Iran is on a rise, there should be a different caring approach for Corona-infected patients in comparison to other patients. Nowadays, health care providers are forced to put themselves at a significant risk because many cases are asymptomatic and they have to care for patients due to their professional status.
To address this issue and reduce medical errors due to incomplete examination, providing enough PPE is very important in all kinds of hospitals (corona or non-corona centers). We also propose that educational courses and enough experiment for triage staffs have a crucial role in decision-making. In this regard, they would be able to handle and triage patients in disasters or disease outbreaks like the case of COVID-19 outbreak. Furthermore, patients' transfer or referral between hospitals from the triage or ED should be confirmed by an emergency medicine physician. This is highly essential as critical and life threatening situations might be missed without a brief examination.

\section{Conclusion}

Although COVID-19 is the main health concern these days, other critical conditions should be taken into account. Stabilizing patients before transferring them between hospitals should be the essential goal of EDs whether the patient is Corona virus infected or not. But before any intervention, considering the safety of healthcare workers must be ensured.

\section{Implications for practice}

The pandemic of COVID-19 has an impact on ED referral. Focusing on low SPO2 and COVID-19 might increase the risk of medical errors in triage.

\section{Authors' contributions}

All authors are contributed in data collection and writing paper.

\section{Ethical issues}

Informed consent was obtained from all patients for publication of the report.

\section{Acknowledgement}

We are indebted to all patients.

\section{References}

1. Velavan TP, Meyer CG. The COVID-19 epidemic. Trop Med Int Health. 2020; 25(3): 278-280. doi: 10.1111/tmi.13383.

2. Millán R, Thomas-Paulose D, Egan DJ, Nusbaum J, Gupta N. Recognizing and managing emerging infectious diseases in the emergency department [digest]. Emerg Med Pract 2018; 20 (Suppl 5): 1-2.

3. Giwa A, Ogedegbe C, Murphy CG. Influenza: diagnosis and management in the emergency department. Emerg Med Pract 2018; 20(12): 1-20.

4. Rahmani Sh. COVID-19 epidemic: What happens to other routine patients admitted in the emergency department? Journal of Emergency Practice and Trauma 2020; 6(2): 5354. doi: 10.34172/jept.2020.19.

5. Zu ZY, Jiang MD, Xu PP, Chen W, Ni QQ, Lu GM, et al. Coronavirus disease 2019 (COVID-19): a perspective from China. Radiology 2020 Feb 21: 200490. doi: 10.1148/ radiol.2020200490. 